\title{
Symmetric duality for a higher-order nondifferentiable multiobjective programming problem
}

\author{
Indira P Debnath', Shiv K Gupta ${ }^{*}$ and Sumit Kumar ${ }^{2}$
}

\section{"Correspondence:}

skgiitr@gmail.com

'Department of Mathematics,

Indian Institute of Technology,

Roorkee, 247 667, India

Full list of author information is

available at the end of the article

\begin{abstract}
In this paper, a pair of Wolfe type higher-order nondifferentiable symmetric dual programs over arbitrary cones has been studied and then well-suited duality relations have been established considering K-F convexity assumptions. An example which satisfies the weak duality relation has also been depicted.
\end{abstract}

MSC: 90C29; 90C30; 49N15

Keywords: symmetric duality; higher-order K-F convexity; multiobjective programming; support function; efficient solutions

\section{Introduction}

Consider the following multiobjective programming problem:

(P) $\quad K$-minimize $f(x)$

$$
\text { subject to } x \in X^{0}=\{x \in S:-g(x) \in C\} \text {, }
$$

where $S \subset R^{n}$ be open, $f: S \rightarrow R^{k}, g: S \rightarrow R^{m}, K$, and $C$ are closed convex pointed cones with nonempty interiors in $R^{k}$ and $R^{m}$, respectively.

Several researchers have studied the duality relations for different dual problems of $(\mathrm{P})$ under various generalized convexity assumptions. Chen [1] considered a pair of symmetric higher-order Mond-Weir type nondifferentiable multiobjecive programming problems and established duality relations under higher-order $F$-convexity assumptions. Later on, Agarwal et al. [2] have filled some of the gap in the work of Chen [1] and proved a strong duality theorem for a Mond-Weir type multiobjective higher-order nondifferentiable symmetric dual program. Khurana [3] considered a pair of Mond-Weir type symmetric dual multiobjective programs over arbitrary cones and established duality results under conepseudoinvex and strongly cone-pseudoinvex assumptions. Later on, Kim and Kim [4] extended the results in Khurana [3] to the nondifferentiable multiobjective symmetric dual problem. Gupta and Jayswal [5] studied the higher-order Mond-Weir type multiobjective symmetric duality over cones using higher-order cone-preinvex and cone-pseudoinvex functions, which further extends some of the results in $[3,6,7]$.

Agarwal et al. [8] formulated a pair of Mond-Weir type nondifferentiable multiobjective higher-order symmetric dual programs over arbitrary cones and established duality

\section{Springer}

(02015 Debnath et al.; licensee Springer. This is an Open Access article distributed under the terms of the Creative Commons Attribution License (http://creativecommons.org/licenses/by/2.0), which permits unrestricted use, distribution, and reproduction in any medium, provided the original work is properly cited. 
theorems under higher-order $K-F$ convexity assumptions. In the recent work of Suneja and Louhan [9], the authors have considered Wolfe and Mond-Weir type differentiable symmetric higher-order dual pairs. The Mond-Weir type model studied in [9] is similar to the problem considered in Gupta and Jayswal [5]. However, the strong duality result in [9] is for arbitrary cones in $R^{k}$ instead of only those cones which contain the nonnegative orthant of $R^{k}$ as considered in [5].

In the present paper, a pair of Wolfe type higher-order multiobjective nondifferentiable symmetric dual program have been formulated and we established weak, strong, and converse duality theorems under $K-F$ convexity assumptions. We also illustrate a nontrivial example of a function which satisfies the weak duality relation.

\section{Definitions and preliminaries}

Let $C_{1} \subseteq R^{n}$ and $C_{2} \subseteq R^{m}$ be closed convex cones with nonempty interiors and let $S_{1}$ and $S_{2}$ be nonempty open sets in $R^{n}$ and $R^{m}$, respectively such that $C_{1} \times C_{2} \subseteq S_{1} \times S_{2}$. For a real valued twice differentiable function $f(x, y)$ defined on $S_{1} \times S_{2}, \nabla_{x} f(x, y)$ denotes the gradient vector of $f$ with respect to $x$ at $(x, y), \nabla_{x x} f(x, y)$ denotes the Hessian matrix with respect to $x$ at $(x, y)$. Similarly, $\nabla_{y} f(x, y), \nabla_{x y} f(x, y)$, and $\nabla_{y y} f(x, y)$ are also defined.

Definition 2.1 [8] A point $\bar{x} \in X^{0}$ is a weak efficient solution of (P) if there exists no $x \in X^{0}$ such that

$$
f(\bar{x})-f(x) \in \operatorname{int} K
$$

Definition 2.2 [5] A point $\bar{x} \in X^{0}$ is an efficient solution of (P) if there exists no $x \in X^{0}$ such that

$$
f(\bar{x})-f(x) \in K \backslash\{0\} .
$$

Definition 2.3 The positive dual cone $K^{+}$of $K$ is defined by

$$
K^{+}=\left\{y: x^{T} y \geqq 0 \text { for all } x \in K\right\} .
$$

Definition 2.4 For all $(x, u) \in S_{1} \times S_{2}$, a functional $F: S_{1} \times S_{2} \times R^{n} \rightarrow R$ is said to be sublinear with respect to the third variable, if

(i) $F\left(x, u ; a_{1}+a_{2}\right) \leqq F\left(x, u ; a_{1}\right)+F\left(x, u ; a_{2}\right)$ for all $a_{1}, a_{2} \in R^{n}$,

(ii) $F(x, u ; \beta a)=\beta F(x, u ; a)$, for all $\beta \in R_{+}$and for all $a \in R^{n}$.

For convenience, we write $F(x, u ; a)=F_{x, u}(a)$.

Definition 2.5 [8] Let $F: S_{1} \times S_{2} \times R^{n} \rightarrow R$ be a sublinear functional with respect to the third variable. Also, let $h_{i}: S_{1} \times R^{n} \rightarrow R, i=1,2, \ldots, k$ be a differentiable function. Then the function $f: S_{1} \times S_{2} \rightarrow R^{k}$ is said to be higher-order $K-F$ convex in the first variable at $u \in S_{1}$ for fixed $v \in S_{2}$ with respect to $h$, such that for $x \in S_{1}, p_{i} \in R^{n}, i=1,2, \ldots, k$,

$$
\begin{aligned}
& \left(f_{1}(x, v)-f_{1}(u, v)-F_{x, u}\left(\nabla_{x} f_{1}(u, v)+\nabla_{p_{1}} h_{1}\left(u, p_{1}\right)\right)-h_{1}\left(u, p_{1}\right)+p_{1}^{T}\left[\nabla_{p_{1}} h_{1}\left(u, p_{1}\right)\right], \ldots,\right. \\
& \left.\quad f_{k}(x, v)-f_{k}(u, v)-F_{x, u}\left(\nabla_{x} f_{k}(u, v)+\nabla_{p_{k}} h_{k}\left(u, p_{k}\right)\right)-h_{k}\left(u, p_{k}\right)+p_{k}^{T}\left[\nabla_{p_{k}} h_{k}\left(u, p_{k}\right)\right]\right) \\
& \in K .
\end{aligned}
$$


Definition 2.6 [10] Let $\varphi$ be a compact convex set in $R^{n}$. The support function of $\varphi$ is defined by

$$
S(x \mid \varphi)=\max \left\{x^{T} y: y \in \varphi\right\} .
$$

The subdifferentiable of $S(x \mid \varphi)$ is given by

$$
\partial S(x \mid \varphi)=\left\{z \in \varphi: z^{T} x=S(x \mid \varphi)\right\} .
$$

For any set $S \subset R^{n}$, the normal cone to $S$ at a point $x \in S$ is defined by

$$
N_{S}(x)=\left\{y \in R^{n}: y^{T}(z-x) \leqq 0 \text { for all } z \in S\right\} .
$$

For each $i=1,2, \ldots, k$, let $f_{i}: S_{1} \times S_{2} \rightarrow R, h_{i}: S_{1} \times S_{2} \times R^{m} \rightarrow R$ and $g_{i}: S_{1} \times S_{2} \times R^{n} \rightarrow R$ be differentiable functions. $p=\left(p_{1}, p_{2}, \ldots, p_{k}\right)$ and $r=\left(r_{1}, r_{2}, \ldots, r_{k}\right)$, for $p_{i} \in R^{m}$ and $r_{i} \in R^{n}$, $i=1,2, \ldots, k . C_{1}^{+}$and $C_{2}^{+}$are the positive dual cones of $C_{1}$ and $C_{2}$, respectively. $D$ and $E$ are the compact convex sets in $R^{n}$ and $R^{m}$, respectively. Also, we use the following notations:

$$
\begin{aligned}
& h(x, y, p)=\left(h_{1}\left(x, y, p_{1}\right), h_{2}\left(x, y, p_{2}\right), \ldots, h_{k}\left(x, y, p_{k}\right)\right), \\
& g(u, v, r)=\left(g_{1}\left(u, v, r_{1}\right), g_{2}\left(u, v, r_{2}\right), \ldots, g_{k}\left(u, v, r_{k}\right)\right), \\
& \nabla_{p} h(x, y, p)=\left(\nabla_{p_{1}} h_{1}\left(x, y, p_{1}\right), \nabla_{p_{2}} h_{2}\left(x, y, p_{2}\right), \ldots, \nabla_{p_{k}} h_{k}\left(x, y, p_{k}\right)\right), \\
& \nabla_{r} g(u, v, r)=\left(\nabla_{r_{1}} g_{1}\left(u, v, r_{1}\right), \nabla_{r_{2}} g_{2}\left(u, v, r_{2}\right), \ldots, \nabla_{r_{k}} g_{k}\left(u, v, r_{k}\right)\right), \\
& p^{T} \nabla_{p} h(x, y, p)=\left(p_{1}^{T} \nabla_{p_{1}} h_{1}\left(x, y, p_{1}\right), p_{2}^{T} \nabla_{p_{2}} h_{2}\left(x, y, p_{2}\right), \ldots, p_{k}^{T} \nabla_{p_{k}} h_{k}\left(x, y, p_{k}\right)\right) \quad \text { and } \\
& r^{T} \nabla_{r} g(u, v, r)=\left(r_{1}^{T} \nabla_{r_{1}} g_{1}\left(u, v, r_{1}\right), r_{2}^{T} \nabla_{r_{2}} g_{2}\left(u, v, r_{2}\right), \ldots, r_{k}^{T} \nabla_{r_{k}} g_{k}\left(u, v, r_{k}\right)\right) .
\end{aligned}
$$

\section{Problem formulation}

Consider the following pair of Wolfe type higher-order nondifferentiable multiobjective symmetric dual programs:

(WHP) $K$-minimize $f(x, y)+h(x, y, p)+S(x \mid D) e-p^{T} \nabla_{p} h(x, y, p)$

$$
-y^{T}\left(\sum_{i=1}^{k} \lambda_{i}\left\{\nabla_{y} f_{i}(x, y)+\nabla_{p_{i}} h_{i}\left(x, y, p_{i}\right)\right\} e\right)
$$

subject to

$$
\begin{aligned}
& -\left(\sum_{i=1}^{k} \lambda_{i}\left\{\nabla_{y} f_{i}(x, y)+\nabla_{p_{i}} h_{i}\left(x, y, p_{i}\right)\right\}-z\right) \in C_{2}^{+}, \\
& \lambda=\left(\lambda_{1}, \lambda_{2}, \ldots, \lambda_{k}\right) \in \operatorname{int} K^{+}, \quad \lambda^{T} e=1, \quad x \in C_{1}, \quad z \in E,
\end{aligned}
$$

(WHD) K-maximize $f(u, v)+g(u, v, r)-S(v \mid E) e-r^{T} \nabla_{r} g(u, v, r)$

$$
-u^{T}\left(\sum_{i=1}^{k} \lambda_{i}\left\{\nabla_{u} f_{i}(u, v)+\nabla_{r_{i}} g_{i}\left(u, v, r_{i}\right)\right\} e\right)
$$

subject to 


$$
\begin{aligned}
& \left(\sum_{i=1}^{k} \lambda_{i}\left\{\nabla_{u} f_{i}(u, v)+\nabla_{r_{i}} g_{i}\left(u, v, r_{i}\right)\right\}+w\right) \in C_{1}^{+}, \\
& \lambda=\left(\lambda_{1}, \lambda_{2}, \ldots, \lambda_{k}\right) \in \operatorname{int} K^{+}, \quad \lambda^{T} e=1, \quad v \in C_{2}, \quad w \in D,
\end{aligned}
$$

where $e=\left(e_{1}, e_{2}, \ldots, e_{k}\right) \in \operatorname{int} K$ is fixed.

Remark 3.1 If $D=\{0\}$ and $E=\{0\}$, then our problems (WHP) and (WHD) become the problem studied in Suneja and Louhan [9].

Next, we will prove weak, strong, and converse duality results between (WHP) and (WHD).

Theorem 3.1 (Weak duality) Let $(x, y, \lambda, z, p)$ and $(u, v, \lambda, w, r)$ be feasible solutions for (WHP) and (WHD), respectively. Assume the following conditions hold:

(I) $f(\cdot, v)+(\cdot)^{T}$ we is higher-order $K-F$ convex at $u$ with respect to $g(u, v, r)$ for fixed $v$,

(II) $-f(x, \cdot)+(\cdot)^{T} z e$ is higher-order $K-G$ convex at $y$ with respect to $-h(x, y, p)$ for fixed $x$,

(III) $R_{+}^{k} \subseteq K$,

where $F: S_{1} \times S_{2} \times R^{n} \rightarrow R$ and $G: S_{1} \times S_{2} \times R^{m} \rightarrow R$ are the sublinear functionals with respect to the third variable and satisfy the following conditions:

$$
\begin{aligned}
& F_{x, u}(a)+u^{T} a \geqq 0 \quad \text { for all } a \in C_{1}^{+}, \\
& G_{v, y}(b)+b^{T} y \geqq 0 \quad \text { for all } b \in C_{2}^{+} .
\end{aligned}
$$

Then

$$
\begin{aligned}
& {\left[f(u, v)+g(u, v, r)-S(v \mid E) e-r^{T} \nabla_{r} g(u, v, r)-u^{T} \sum_{i=1}^{k} \lambda_{i}\left\{\nabla_{u} f(u, v)+\nabla_{r_{i}} g_{i}\left(u, v, r_{i}\right)\right\} e\right]} \\
& -\left[f(x, y)+h(x, y, p)+S(x \mid D) e-p^{T} \nabla_{p} h(x, y, p)\right. \\
& \left.-y^{T} \sum_{i=1}^{k} \lambda_{i}\left\{\nabla_{y} f(x, y)+\nabla_{p_{i}} h_{i}\left(x, y, p_{i}\right)\right\} e\right] \\
& \notin K \backslash\{0\} .
\end{aligned}
$$

Proof We shall obtain the proof by contradiction. Let (5) not hold. Then

$$
\begin{aligned}
& {\left[f(u, v)+g(u, v, r)-S(v \mid E) e-r^{T} \nabla_{r} g(u, v, r)-u^{T} \sum_{i=1}^{k} \lambda_{i}\left\{\nabla_{u} f(u, v)+\nabla_{r_{i}} g_{i}\left(u, v, r_{i}\right)\right\} e\right]} \\
& -\left[f(x, y)+h(x, y, p)+S(x \mid D) e-p^{T} \nabla_{p} h(x, y, p)\right. \\
& \left.\quad-y^{T} \sum_{i=1}^{k} \lambda_{i}\left\{\nabla_{y} f(x, y)+\nabla_{p_{i}} h_{i}\left(x, y, p_{i}\right)\right\} e\right] \\
& \in K \backslash\{0\} .
\end{aligned}
$$


It follows from $\lambda \in \operatorname{int} K^{+}$and $\lambda^{T} e=1$ that

$$
\begin{aligned}
& {\left[\sum_{i=1}^{k} \lambda_{i}\left\{f_{i}(u, v)+g_{i}\left(u, v, r_{i}\right)-r_{i}^{T} \nabla_{r_{i}} g_{i}\left(u, v, r_{i}\right)\right\}-S(v \mid E)\right.} \\
& \left.\quad-u^{T} \sum_{i=1}^{k} \lambda_{i}\left\{\nabla_{u} f_{i}(u, v)+\nabla_{r_{i}} g_{i}\left(u, v, r_{i}\right)\right\}\right] \\
& \quad-\left[\sum_{i=1}^{k} \lambda_{i}\left\{f_{i}(x, y)+h_{i}\left(x, y, p_{i}\right)-p_{i}^{T} \nabla_{p_{i}} h_{i}\left(x, y, p_{i}\right)\right\}\right. \\
& \left.\quad+S(x \mid D)-y^{T} \sum_{i=1}^{k} \lambda_{i}\left\{\nabla_{y} f_{i}(x, y)+\nabla_{p_{i}} h_{i}\left(x, y, p_{i}\right)\right\}\right]>0 .
\end{aligned}
$$

Now, since $f(\cdot, v)+(\cdot)^{T}$ we is higher-order $K-F$ convex at $u$ with respect to $g(u, v, r)$ for fixed $v$, we get

$$
\begin{aligned}
& \left(f_{1}(x, v)+x^{T} w e_{1}-f_{1}(u, v)-u^{T} w e_{1}-F_{x, u}\left[\nabla_{u} f_{1}(u, v)+w e_{1}+\nabla_{r_{1}} g_{1}\left(u, v, r_{1}\right)\right]\right. \\
& \quad-g_{1}\left(u, v, r_{1}\right)+r_{1}^{T} \nabla_{r_{1}} g_{1}\left(u, v, r_{1}\right), \ldots, f_{k}(x, v)+x^{T} w e_{k}-f_{k}(u, v)-u^{T} w e_{k} \\
& \left.\quad-F_{x, u}\left[\nabla_{u} f_{k}(u, v)+w e_{k}+\nabla_{r_{k}} g_{k}\left(u, v, r_{k}\right)\right]-g_{k}\left(u, v, r_{k}\right)+r_{k}^{T} \nabla_{r_{k}} g_{k}\left(u, v, r_{k}\right)\right) \in K .
\end{aligned}
$$

Using $\lambda \in \operatorname{int} K^{+}$and $\lambda^{T} e=1$, it follows that

$$
\begin{aligned}
& \sum_{i=1}^{k} \lambda_{i}\left\{f_{i}(x, v)-f_{i}(u, v)-g_{i}\left(u, v, r_{i}\right)+r_{i}^{T} \nabla_{r_{i}} g_{i}\left(u, v, r_{i}\right)\right\}+x^{T} w-u^{T} w \\
& \quad \geqq \sum_{i=1}^{k} \lambda_{i} F_{x, u}\left[\left(\nabla_{u} f_{i}(u, v)+\nabla_{r_{i}} g_{i}\left(u, v, r_{i}\right)\right)+w e_{i}\right] .
\end{aligned}
$$

Since $\lambda \in \operatorname{int} K^{+} \subseteq \operatorname{int} R_{+}^{k}$ (by hypothesis (III)), hence $\lambda>0$. Therefore, using (2) and sublinearity of $F$ in the above expression, we obtain

$$
\begin{aligned}
& \sum_{i=1}^{k} \lambda_{i}\left\{f_{i}(x, v)-f_{i}(u, v)-g_{i}\left(u, v, r_{i}\right)+r_{i}^{T} \nabla_{r_{i}} g_{i}\left(u, v, r_{i}\right)\right\}+x^{T} w-u^{T} w \\
& \quad \geqq \sum_{i=1}^{k} F_{x, u}\left[\lambda_{i}\left(\nabla_{u} f_{i}(u, v)+\nabla_{r_{i}} g_{i}\left(u, v, r_{i}\right)\right)+w\right] .
\end{aligned}
$$

It follows from (A) and the dual constraint (3) that

$$
\begin{aligned}
& \sum_{i=1}^{k} \lambda_{i}\left\{f_{i}(x, v)-f_{i}(u, v)-g_{i}\left(u, v, r_{i}\right)+r_{i}^{T} \nabla_{r_{i}} g_{i}\left(u, v, r_{i}\right)\right\}+x^{T} w-u^{T} w \\
& \quad \geqq-u^{T}\left(\sum_{i=1}^{k} \lambda_{i}\left[\nabla_{u} f_{i}(u, v)+\nabla_{r_{i}} g_{i}\left(u, v, r_{i}\right)\right]+w\right)
\end{aligned}
$$

for $a=\left(\sum_{i=1}^{k} \lambda_{i}\left[\nabla_{u} f_{i}(u, v)+\nabla_{r_{i}} g_{i}\left(u, v, r_{i}\right)\right]+w\right) \in C_{1}^{+}$. 
Similarly, using hypothesis (II), (B), $\lambda>0,(1),(2)$, and sublinearity of $G$, we obtain

$$
\begin{aligned}
& \sum_{i=1}^{k} \lambda_{i}\left\{f_{i}(x, y)-f_{i}(x, v)+h_{i}\left(x, y, p_{i}\right)-p_{i}^{T} \nabla_{p_{i}} h_{i}\left(x, y, p_{i}\right)\right\}+v^{T} z-y^{T} z \\
& \quad \geqq y^{T}\left(\sum_{i=1}^{k} \lambda_{i}\left[\nabla_{y} f_{i}(x, y)+\nabla_{p_{i}} h_{i}\left(x, y, p_{i}\right)\right]-z\right)
\end{aligned}
$$

for $b=-\left(\sum_{i=1}^{k} \lambda_{i}\left[\nabla_{y} f_{i}(x, y)+\nabla_{p_{i}} h_{i}\left(x, y, p_{i}\right)\right]-z\right) \in C_{2}^{+}$.

Now, adding (7) and (8), we have

$$
\begin{aligned}
\sum_{i=1}^{k} \lambda_{i}\left\{f_{i}(x, y)-f_{i}(u, v)-g_{i}\left(u, v, r_{i}\right)+r_{i}^{T} \nabla_{r_{i}} g_{i}\left(u, v, r_{i}\right)\right. \\
\left.\quad+h_{i}\left(x, y, p_{i}\right)-p_{i}^{T} \nabla_{p_{i}} h_{i}\left(x, y, p_{i}\right)\right\}+x^{T} w+v^{T} z \\
\geqq-u^{T} \sum_{i=1}^{k} \lambda_{i}\left[\nabla_{u} f_{i}(u, v)+\nabla_{r_{i}} g_{i}\left(u, v, r_{i}\right)\right] \\
\quad+y^{T} \sum_{i=1}^{k} \lambda_{i}\left[\nabla_{y} f_{i}(x, y)+\nabla_{p_{i}} h_{i}\left(x, y, p_{i}\right)\right] .
\end{aligned}
$$

Finally, it follows from $x^{T} w \leqq S(x \mid D)$ and $v^{T} z \leqq S(v \mid E)$ that

$$
\begin{aligned}
\sum_{i=1}^{k} \lambda_{i}\left\{f_{i}(x, y)+h_{i}\left(x, y, p_{i}\right)-p_{i}^{T} \nabla_{p_{i}} h_{i}\left(x, y, p_{i}\right)\right\} & \\
& +S(x \mid D)-y^{T} \sum_{i=1}^{k} \lambda_{i}\left[\nabla_{y} f_{i}(x, y)+\nabla_{p_{i}} h_{i}\left(x, y, p_{i}\right)\right] \\
\geqq & \sum_{i=1}^{k} \lambda_{i}\left\{f_{i}(u, v)+g_{i}\left(u, v, r_{i}\right)-r_{i}^{T} \nabla_{r_{i}} g_{i}\left(u, v, r_{i}\right)\right\} \\
& -S(v \mid E)-u^{T} \sum_{i=1}^{k} \lambda_{i}\left[\nabla_{u} f_{i}(u, v)+\nabla_{r_{i}} g_{i}\left(u, v, r_{i}\right)\right],
\end{aligned}
$$

which contradicts (6). Hence the result.

Example 3.1 Let $k=2, n=m=1$. Let $S_{1}=S_{2}=R_{+}=\{x \in R: x \geqq 0\}, C_{1}=C_{2}=R_{+}$, and $K=\left\{(x, y) \in R^{2}: x \geqq 0, y \geqq-x\right\}$.

Then $C_{1}^{+}=C_{2}^{+}=R_{+}$and $K^{+}=\left\{(x, y) \in R^{2}: x \geqq 0, x \geqq y\right\}$. Obviously, $R_{+}^{2} \subseteq K$.

Let $f: S_{1} \times S_{2} \rightarrow R, g: S_{1} \times S_{2} \times R^{n} \rightarrow R$ and $h: S_{1} \times S_{2} \times R^{m} \rightarrow R$ be defined as

$$
f(x, y)=\left(x^{2}-y^{2}, x^{2}\right), \quad g(u, v, r)=\left(-r_{1} u,-r_{2} u\right) \quad \text { and } \quad h(x, y, p)=\left(p_{1} y, p_{2} y\right) .
$$

Let $D=[0,1]$ and $E=\{0\}$. Then $S(x \mid D)=\frac{x+|x|}{2}$ and $S(\nu \mid E)=0$. Suppose $\left(e_{1}, e_{2}\right)=(1,1) \in$ int $K$. Also, suppose the sublinear functionals $F$ and $G$ are defined as

$$
F_{x, u}(a)=x^{T} a \quad \text { and } \quad G_{v, y}(b)=b^{T} v .
$$


Now, substituting the above defined expressions in the problems (WHP) and (WHD), we get

(EP) $\quad K$-minimize $\left(x^{2}-y^{2}+\frac{x+|x|}{2}+\left(\lambda_{1}-\lambda_{2}\right) y^{2}, x^{2}+\frac{x+|x|}{2}+\left(\lambda_{1}-\lambda_{2}\right) y^{2}\right)$ subject to

$$
\begin{aligned}
& \left(\lambda_{1}-\lambda_{2}\right) y \geqq 0, \\
& \lambda_{1}>0, \quad \lambda_{1}-\lambda_{2}>0, \quad \lambda_{1}+\lambda_{2}=1, \quad x \geqq 0,
\end{aligned}
$$

(ED) $K$-maximize $\left(u^{2}-v^{2}-\left(\lambda_{1}+\lambda_{2}\right) u^{2}, u^{2}-\left(\lambda_{1}+\lambda_{2}\right) u^{2}\right)$

subject to

$$
\begin{aligned}
& \left(\lambda_{1}+\lambda_{2}\right) u+w \geqq 0, \\
& \lambda_{1}>0, \quad \lambda_{1}-\lambda_{2}>0, \quad \lambda_{1}+\lambda_{2}=1, \quad v \geqq 0, \quad w \in[0,1] .
\end{aligned}
$$

Now, we shall show that for the primal-dual pair (EP) and (ED), the hypotheses of Theorem 3.1 hold.

(A.1) $f(\cdot, v)+(\cdot)^{T} w e$ is higher-order $K-F$ convex at $u=0 \in S_{1}$ with respect to $g(u, v, r)$ for fixed $v$ and for all $x \in S_{1}, r_{1}, r_{2} \in R$, and we have

$$
\begin{aligned}
\left(f_{1}(x, v)\right. & +x^{T} w e_{1}-f_{1}(u, v)-u^{T} w e_{1}-F_{x, u}\left[\nabla_{u} f_{1}(u, v)+w e_{1}+\nabla_{r_{1}} g_{1}\left(u, v, r_{1}\right)\right] \\
& -g_{1}\left(u, v, r_{1}\right)+r_{1}^{T} \nabla_{r_{1}} g_{1}\left(u, v, r_{1}\right), f_{2}(x, v)+x^{T} w e_{2}-f_{2}(u, v)-u^{T} w e_{2} \\
& -F_{x, u}\left[\nabla_{u} f_{2}(u, v)+w e_{2}+\nabla_{r_{2}} g_{2}\left(u, v, r_{2}\right)\right] \\
& \left.-g_{2}\left(u, v, r_{2}\right)+r_{2}^{T} \nabla_{r_{2}} g_{2}\left(u, v, r_{2}\right)\right) \\
= & \left(x^{2}, x^{2}\right) \in K .
\end{aligned}
$$

(A.2) $-f(x, \cdot)+(\cdot)^{T} z e$ is higher-order $K-G$ convex at $y=0 \in S_{2}$ with respect to $-h(x, y, p)$ for fixed $x$ and for all $v \in S_{2}, p_{1}, p_{2} \in R$, and we have

$$
\begin{aligned}
& \left(-f_{1}(x, v)+v^{T} z e_{1}+f_{1}(x, y)-y^{T} z e_{1}-G_{v, y}\left[-\nabla_{y} f_{1}(x, y)+z e_{1}-\nabla_{p_{1}} h_{1}\left(x, y, p_{1}\right)\right]\right. \\
& \quad+h_{1}\left(x, y, p_{1}\right)-p_{1}^{T} \nabla_{p_{1}} h_{1}\left(x, y, p_{1}\right),-f_{2}(x, v)+v^{T} z e_{2}+f_{2}(x, y)-y^{T} z e_{2} \\
& \quad-G_{v, y}\left[-\nabla_{y} f_{2}(x, y)+z e_{2}-\nabla_{p_{2}} h_{2}\left(x, y, p_{2}\right)\right] \\
& \left.\quad+h_{2}\left(x, y, p_{2}\right)-p_{2}^{T} \nabla_{p_{2}} h_{2}\left(x, y, p_{2}\right)\right) \\
& =\left(v^{2}, 0\right) \in K .
\end{aligned}
$$

(A.3)

$$
\begin{aligned}
& F_{x, u}(a)+u^{T} a=(x+u)^{T} a \geqq 0, \quad \forall a \in C_{1}^{+} \text {and } \forall x, u \in S_{1}, \\
& G_{v, y}(b)+b^{T} y=(v+y)^{T} b \geqq 0, \quad \forall b \in C_{2}^{+} \text {and } \forall v, y \in S_{2} .
\end{aligned}
$$

The points $\left(x, y, \lambda_{1}, \lambda_{2}, z, p_{1}, p_{2}\right)=\left(1,0, \frac{3}{4}, \frac{1}{4}, 0,1,1\right)$ and $\left(u, v, \lambda_{1}, \lambda_{2}, w, r_{1}, r_{2}\right)=\left(0,1, \frac{3}{4}, \frac{1}{4}, 1\right.$, $1,1)$ are feasible for the problems (EP) and (ED), respectively. These feasible points do 
satisfy the result of the weak duality theorem since

$$
\begin{aligned}
& {\left[f(u, v)+g(u, v, r)-S(v \mid E) e-r^{T} \nabla_{r} g(u, v, r)-u^{T} \sum_{i=1}^{k} \lambda_{i}\left\{\nabla_{u} f(u, v)+\nabla_{r_{i}} g_{i}\left(u, v, r_{i}\right)\right\} e\right]} \\
& \quad-\left[f(x, y)+h(x, y, p)+S(x \mid D) e-p^{T} \nabla_{p} h(x, y, p)\right. \\
& \left.\quad-y^{T} \sum_{i=1}^{k} \lambda_{i}\left\{\nabla_{y} f(x, y)+\nabla_{p_{i}} h_{i}\left(x, y, p_{i}\right)\right\} e\right] \\
& =(-3,-2) \notin K \backslash\{0\} .
\end{aligned}
$$

Theorem 3.2 (Strong duality) Let $(\bar{x}, \bar{y}, \bar{\lambda}, \bar{z}, \bar{p})$ be a weak efficient solution of (WHD). Let

(I) the Hessian matrix $\nabla_{p_{i} p_{i}} h_{i}\left(\bar{x}, \bar{y}, \bar{p}_{i}\right)$ for all $i=1,2, \ldots, k$ be positive or negative definite;

(II) $\bar{p}_{i} \neq 0$, for some $i \in\{1,2, \ldots, k\}$ imply that $\sum_{i=1}^{k} \xi_{i} \nabla_{y y} f_{i}(\bar{x}, \bar{y}) \bar{p}_{i} \neq 0$ for all $\xi \in K^{+}$;

(III) $\sum_{i=1}^{k} \xi_{i} \nabla_{y y} f_{i}(\bar{x}, \bar{y}) \bar{p}_{i} \notin \operatorname{span}\left\{\nabla_{y} f_{i}(\bar{x}, \bar{y})+\nabla_{y} h_{i}\left(\bar{x}, \bar{y}, \bar{p}_{i}\right), \nabla_{y} f_{i}(\bar{x}, \bar{y})+\nabla_{p_{i}} h_{i}\left(\bar{x}, \bar{y}, \bar{p}_{i}\right)\right.$, $i=1,2, \ldots, k\} \backslash\{0\}$, for all $\xi \in K^{+}$;

(IV) the set of vectors $\left\{\nabla_{y} f_{i}(\bar{x}, \bar{y}): i=1,2, \ldots, k\right\}$ be linearly independent;

(V) $\nabla_{y} h_{i}(\bar{x}, \bar{y}, 0)=0=\nabla_{p_{i}} h_{i}(\bar{x}, \bar{y}, 0), h_{i}(\bar{x}, \bar{y}, 0)=g_{i}(\bar{x}, \bar{y}, 0), \nabla_{x} h_{i}(\bar{x}, \bar{y}, 0)=\nabla_{r_{i}} g_{i}(\bar{x}, \bar{y}, 0)$, for all $i=\{1,2, \ldots, k\}$.

Then

(I) there exists $\bar{w} \in D$ such that $(\bar{x}, \bar{y}, \bar{\lambda}, \bar{w}, \bar{r}=0)$ is feasible for (WHD) and

(II) the objective values of (WHP) and (WHD) are equal.

Also, if the hypotheses of Theorem 3.1 are satisfied for all feasible solutions of (WHP) and (WHD), then $(\bar{x}, \bar{y}, \bar{\lambda}, \bar{w}, \bar{r}=0)$ is an efficient solution for (WHD).

Proof Since $(\bar{x}, \bar{y}, \bar{\lambda}, \bar{z}, \bar{p})$ is a weak efficient solution for (WHP), by the Fritz John necessary optimality conditions [11], there exist $\alpha=\left(\alpha_{1}, \alpha_{2}, \ldots, \alpha_{k}\right) \in K^{+}, \beta \in C_{2}$, and $\eta \in R$ such that

$$
\begin{aligned}
& {\left[\sum_{i=1}^{k} \alpha_{i}\left(\nabla_{x} f_{i}(\bar{x}, \bar{y})+\nabla_{x} h_{i}\left(\bar{x}, \bar{y}, \bar{p}_{i}\right)\right)+\left(\alpha^{T} e\right) \gamma+\sum_{i=1}^{k} \bar{\lambda}_{i}\left\{\beta-\left(\alpha^{T} e\right) \bar{y}\right\} \nabla_{x y} f_{i}(\bar{x}, \bar{y})\right.} \\
& \left.\quad+\sum_{i=1}^{k} \nabla_{p_{i} x} h_{i}\left(\bar{x}, \bar{y}, \bar{p}_{i}\right)\left(\bar{\lambda}_{i} \beta-\bar{\lambda}_{i}\left(\alpha^{T} e\right) \bar{y}-\alpha_{i} \bar{p}_{i}\right)\right]^{T}(x-\bar{x}) \geqq 0 \quad \text { for all } x \in C_{1}, \\
& \sum_{i=1}^{k} \alpha_{i}\left[\nabla_{y} f_{i}(\bar{x}, \bar{y})+\nabla_{y} h_{i}\left(\bar{x}, \bar{y}, \bar{p}_{i}\right)\right]-\sum_{i=1}^{k}\left(\alpha^{T} e\right) \bar{\lambda}_{i}\left\{\nabla_{y} f_{i}(\bar{x}, \bar{y})+\nabla_{p_{i}} h_{i}\left(\bar{x}, \bar{y}, \bar{p}_{i}\right)\right\} \\
& \quad+\sum_{i=1}^{k} \bar{\lambda}_{i} \nabla_{y y} f_{i}(\bar{x}, \bar{y})\left(\beta-\left(\alpha^{T} e\right) \bar{y}\right) \\
& \quad+\sum_{i=1}^{k} \nabla_{p_{i} y} h_{i}\left(\bar{x}, \bar{y}, \bar{p}_{i}\right)\left(\bar{\lambda}_{i} \beta-\bar{\lambda}_{i}\left(\alpha^{T} e\right) \bar{y}-\alpha_{i} \bar{p}_{i}\right)=0, \\
& \left\{\beta-\left(\alpha^{T} e\right) \bar{y}\right\}^{T}\left[\nabla_{y} f_{i}(\bar{x}, \bar{y})+\nabla_{p_{i}} h_{i}\left(\bar{x}, \bar{y}, \bar{p}_{i}\right)\right]+\eta e_{i}=0, \quad i=1,2, \ldots, k, \\
& \nabla_{p_{i} p_{i}} h_{i}\left(\bar{x}, \bar{y}, \bar{p}_{i}\right)\left(\bar{\lambda}_{i} \beta-\bar{\lambda}_{i}\left(\alpha^{T} e\right) \bar{y}-\alpha_{i} \bar{p}_{i}\right)=0, \quad i=1,2, \ldots, k,
\end{aligned}
$$




$$
\begin{aligned}
& \beta^{T}\left(\sum_{i=1}^{k} \bar{\lambda}_{i}\left\{\nabla_{y} f_{i}(\bar{x}, \bar{y})+\nabla_{p_{i}} h_{i}\left(\bar{x}, \bar{y}, \bar{p}_{i}\right)\right\}-\bar{z}\right)=0, \quad i=1,2, \ldots, k, \\
& \eta^{T}\left(\bar{\lambda}^{T} e-1\right)=0, \\
& \beta \in N_{E}(\bar{z}) \\
& \gamma \in D, \quad \gamma^{T} \bar{x}=S(\bar{x} \mid D), \\
& (\alpha, \beta, \eta) \neq 0 .
\end{aligned}
$$

Now, hypothesis (I) and (12) imply that

$$
\bar{\lambda}_{i} \beta-\bar{\lambda}_{i}\left(\alpha^{T} e\right) \bar{y}-\alpha_{i} \bar{p}_{i}=0, \quad i=1,2, \ldots, k .
$$

Using (18) in (10), we have

$$
\begin{aligned}
\sum_{i=1}^{k} \alpha_{i} \nabla_{y y} f_{i}(\bar{x}, \bar{y}) \bar{p}_{i}= & \left(\alpha^{T} e\right) \sum_{i=1}^{k} \bar{\lambda}_{i}\left(\nabla_{y} f_{i}(\bar{x}, \bar{y})+\nabla_{p_{i}} h_{i}\left(\bar{x}, \bar{y}, \bar{p}_{i}\right)\right) \\
& -\sum_{i=1}^{k} \alpha_{i}\left(\nabla_{y} f_{i}(\bar{x}, \bar{y})+\nabla_{y} h_{i}\left(\bar{x}, \bar{y}, \bar{p}_{i}\right)\right)
\end{aligned}
$$

which yields

$$
\begin{aligned}
\sum_{i=1}^{k} \alpha_{i} \nabla_{y y} f_{i}(\bar{x}, \bar{y}) \bar{p}_{i} \in & \operatorname{span}\left\{\nabla_{y} f_{i}(\bar{x}, \bar{y})+\nabla_{y} h_{i}\left(\bar{x}, \bar{y}, \bar{p}_{i}\right), \nabla_{y} f_{i}(\bar{x}, \bar{y})\right. \\
& \left.+\nabla_{p_{i}} h_{i}\left(\bar{x}, \bar{y}, \bar{p}_{i}\right), i=1,2, \ldots, k\right\} .
\end{aligned}
$$

Now, we claim that $\bar{p}_{i}=0$ for all $i=1,2, \ldots, k$. On the contrary, suppose that for some $i \in\{1,2, \ldots, k\}, \bar{p}_{i} \neq 0$, then using hypothesis (II), we have

$$
\sum_{i=1}^{k} \alpha_{i} \nabla_{y y} f_{i}(\bar{x}, \bar{y}) \bar{p}_{i} \neq 0 .
$$

This contradicts hypothesis (III) (by (20) and (21)). Hence,

$$
\bar{p}_{i}=0 \quad \text { for all } i=1,2, \ldots, k \text {. }
$$

Using (22) in (18), we have $\bar{\lambda}_{i} \beta=\bar{\lambda}_{i}\left(\alpha^{T} e\right) \bar{y}, i=1,2, \ldots, k$.

Since $\bar{\lambda} \in \operatorname{int} K^{+}, \bar{\lambda}_{i} \neq 0$ for at least one $i$,

$$
\beta=\left(\alpha^{T} e\right) \bar{y}
$$

It follows from (11) and (23) that $\eta e_{i}=0, i=1,2, \ldots, k$, which from $e \in \operatorname{int} K$ implies $\eta=0$.

From (19), (22), and hypothesis (V), we get

$$
\sum_{i=1}^{k}\left\{\alpha_{i}-\left(\alpha^{T} e\right) \bar{\lambda}_{i}\right\} \nabla_{y} f_{i}(\bar{x}, \bar{y})=0
$$


which from hypothesis (IV) yields

$$
\alpha=\left(\alpha^{T} e\right) \bar{\lambda}
$$

Now, if $\alpha=0$, then $\alpha^{T} e=0$. Therefore, from (23), we get $\beta=0$ and hence, $(\alpha, \beta, \eta)=0$. This contradicts (17). Thus $\alpha \neq 0$. Since $\alpha \in K^{+}$and $e \in \operatorname{int} K$, we have

$$
\alpha^{T} e>0
$$

From (23) and (25), we obtain

$$
\bar{y}=\frac{\beta}{\left(\alpha^{T} e\right)} \in C_{2} .
$$

Further, using inequalities (18), (23)-(25) in (9), we obtain

$$
\left[\sum_{i=1}^{k} \bar{\lambda}_{i}\left\{\nabla_{x} f_{i}(\bar{x}, \bar{y})+\nabla_{x} h_{i}\left(\bar{x}, \bar{y}, \bar{p}_{i}\right)\right\}+\gamma\right]^{T}(x-\bar{x}) \geqq 0 \quad \text { for all } x \in C_{1} .
$$

For $\bar{r}=0$, it follows from (22) and hypothesis (V) that

$$
\left[\sum_{i=1}^{k} \bar{\lambda}_{i}\left\{\nabla_{x} f_{i}(\bar{x}, \bar{y})+\nabla_{r_{i}} g_{i}\left(\bar{x}, \bar{y}, \bar{r}_{i}\right)\right\}+\gamma\right]^{T}(x-\bar{x}) \geqq 0 .
$$

Let $x \in C_{1}$. Then $\bar{x}+x \in C_{1}$ and hence from (26), we have

$$
\left[\sum_{i=1}^{k} \bar{\lambda}_{i}\left\{\nabla_{x} f_{i}(\bar{x}, \bar{y})+\nabla_{r_{i}} g_{i}\left(\bar{x}, \bar{y}, \bar{r}_{i}\right)\right\}+\gamma\right]^{T} x \geqq 0 \quad \text { for all } x \in C_{1} .
$$

Therefore, $\left[\sum_{i=1}^{k} \bar{\lambda}_{i}\left\{\nabla_{x} f_{i}(\bar{x}, \bar{y})+\nabla_{r_{i}} g_{i}\left(\bar{x}, \bar{y}, \bar{r}_{i}\right)\right\}+\gamma\right] \in C_{1}^{+}$.

Thus, $(\bar{x}, \bar{y}, \bar{\lambda}, \gamma=\bar{w}, \bar{r}=0)$ is a feasible solution for the dual problem.

Consider $x=0$ and $x=2 \bar{x}$ in (26), we get

$$
\left[\sum_{i=1}^{k} \bar{\lambda}_{i}\left\{\nabla_{x} f_{i}(\bar{x}, \bar{y})+\nabla_{r_{i}} g_{i}\left(\bar{x}, \bar{y}, \bar{r}_{i}\right)\right\}+\gamma\right]^{T} \bar{x}=0,
$$

which implies that

$$
\bar{x}^{T} \sum_{i=1}^{k} \bar{\lambda}_{i}\left\{\nabla_{x} f_{i}(\bar{x}, \bar{y})+\nabla_{r_{i}} g_{i}\left(\bar{x}, \bar{y}, \bar{r}_{i}\right)\right\}=-\bar{x}^{T} \gamma=-S(\bar{x} \mid D) .
$$

Now, (15) and (23) yield $\left(\alpha^{T} e\right) \bar{y} \in N_{E}(\bar{z})$. Since $\alpha^{T} e>0, \bar{y} \in N_{E}(\bar{z})$.

Again as $E$ is a compact convex set in $R^{m}, \bar{y}^{T} \bar{z}=S(\bar{y} \mid E)$.

Further, (13), (23), and (25) yield

$$
\bar{y}^{T} \sum_{i=1}^{k} \bar{\lambda}_{i}\left\{\nabla_{y} f_{i}(\bar{x}, \bar{y})+\nabla_{p_{i}} h_{i}\left(\bar{x}, \bar{y}, \bar{p}_{i}\right)\right\}=\bar{y}^{T} \bar{z}=S(\bar{y} \mid E) .
$$


By hypothesis (V) for $\bar{r}=0$, (22), (27)-(28), we obtain

$$
\begin{aligned}
& f(\bar{x}, \bar{y})+h(\bar{x}, \bar{y}, \bar{p})+S(\bar{x} \mid D) e-\bar{p}^{T} \nabla_{p} h(\bar{x}, \bar{y}, \bar{p})-\bar{y}^{T} \sum_{i=1}^{k} \bar{\lambda}_{i}\left\{\nabla_{y} f_{i}(\bar{x}, \bar{y})+\nabla_{p_{i}} h_{i}\left(\bar{x}, \bar{y}, \bar{p}_{i}\right)\right\} e \\
& \quad=f(\bar{x}, \bar{y})+g(\bar{x}, \bar{y}, \bar{r})-S(\bar{y} \mid E) e-\bar{r}^{T} \nabla_{r} g(\bar{x}, \bar{y}, \bar{r})-\bar{x}^{T} \sum_{i=1}^{k} \bar{\lambda}_{i}\left\{\nabla_{x} f_{i}(\bar{x}, \bar{y})+\nabla_{r_{i}} g_{i}\left(\bar{x}, \bar{y}, \bar{r}_{i}\right)\right\} e .
\end{aligned}
$$

Hence, the two objective values are equal.

Now, let $(\bar{x}, \bar{y}, \bar{\lambda}, \bar{w}, \bar{r}=0)$ be not an efficient solution of (WHD), then there exists a point $(\hat{u}, \hat{v}, \bar{\lambda}, \hat{w}, \hat{r})$ feasible for (WHD) such that

$$
\begin{aligned}
& {\left[f(\hat{u}, \hat{v})+g(\hat{u}, \hat{v}, \hat{r})-S(\hat{v} \mid E) e-\hat{r}^{T} \nabla_{r} g(\hat{u}, \hat{v}, \hat{r})-\hat{u}^{T} \sum_{i=1}^{k} \bar{\lambda}_{i}\left\{\nabla_{u} f_{i}(\hat{u}, \hat{v})+\nabla_{r_{i}} g_{i}\left(\hat{u}, \hat{v}, \hat{r}_{i}\right)\right\} e\right]} \\
& -\left[f(\bar{x}, \bar{y})+g(\bar{x}, \bar{y}, \bar{r})-S(\bar{y} \mid E) e-\bar{r}^{T} \nabla_{r} g(\bar{x}, \bar{y}, \bar{r})\right. \\
& \left.\quad-\bar{x}^{T} \sum_{i=1}^{k} \bar{\lambda}_{i}\left\{\nabla_{x} f_{i}(\bar{x}, \bar{y})+\nabla_{r_{i}} g_{i}\left(\bar{x}, \bar{y}, \bar{r}_{i}\right)\right\} e\right] \\
& \in K \backslash\{0\} .
\end{aligned}
$$

From (27), (28), and hypothesis (V) for $\bar{r}=0$ and $\bar{p}=0$, we obtain

$$
\begin{aligned}
& {\left[f(\hat{u}, \hat{v})+g(\hat{u}, \hat{v}, \hat{r})-S(\hat{v} \mid E) e-\hat{r}^{T} \nabla_{r} g(\hat{u}, \hat{v}, \hat{r})-\hat{u}^{T} \sum_{i=1}^{k} \bar{\lambda}_{i}\left\{\nabla_{u} f_{i}(\hat{u}, \hat{v})+\nabla_{r_{i}} g_{i}\left(\hat{u}, \hat{v}, \hat{r}_{i}\right)\right\} e\right]} \\
& -\left[f(\bar{x}, \bar{y})+h(\bar{x}, \bar{y}, \bar{p})+S(\bar{x} \mid D) e-\bar{p}^{T} \nabla_{p} h(\bar{x}, \bar{y}, \bar{p})\right. \\
& \left.\quad-\bar{y}^{T} \sum_{i=1}^{k} \bar{\lambda}_{i}\left\{\nabla_{y} f_{i}(\bar{x}, \bar{y})+\nabla_{p_{i}} h_{i}\left(\bar{x}, \bar{y}, \bar{p}_{i}\right)\right\} e\right] \\
& \in K \backslash\{0\},
\end{aligned}
$$

which contradicts Theorem 3.1. Hence, $(\bar{x}, \bar{y}, \bar{\lambda}, \bar{w}, \bar{r}=0)$ is the efficient solution of (WHD).

Theorem 3.3 (Converse duality) Let $(\bar{u}, \bar{v}, \bar{\lambda}, \bar{w}, \bar{r})$ be a weak efficient solution of (WHP). Let

(I) the Hessian matrix $\nabla_{r_{i} r_{i}} g_{i}\left(\bar{u}, \bar{v}, \bar{r}_{i}\right)$ for all $i=1,2, \ldots, k$ be positive or negative definite;

(II) $\bar{r}_{i} \neq 0$, for some $i \in\{1,2, \ldots, k\}$ implies that $\sum_{i=1}^{k} \xi_{i} \nabla_{u u} f_{i}(\bar{u}, \bar{v}) \bar{r}_{i} \neq 0$ for all $\xi \in K^{+}$;

(III) $\sum_{i=1}^{k} \xi_{i} \nabla_{u u} f_{i}(\bar{u}, \bar{v}) \bar{r}_{i} \notin \operatorname{span}\left\{\nabla_{u} f_{i}(\bar{u}, \bar{v})+\nabla_{u} g_{i}\left(\bar{u}, \bar{v}, \bar{r}_{i}\right), \nabla_{u} f_{i}(\bar{u}, \bar{v})+\nabla_{r_{i}} g_{i}\left(\bar{u}, \bar{v}, \bar{r}_{i}\right)\right.$, $i=1,2, \ldots, k\} \backslash\{0\}$, for all $\xi \in K^{+}$;

(IV) the set of vectors $\left\{\nabla_{u} f_{i}(\bar{u}, \bar{v}): i=1,2, \ldots, k\right\}$ be linearly independent;

(V) $\nabla_{u} g_{i}(\bar{u}, \bar{v}, 0)=0=\nabla_{r_{i}} g_{i}(\bar{u}, \bar{v}, 0), g_{i}(\bar{u}, \bar{v}, 0)=h_{i}(\bar{u}, \bar{v}, 0), \nabla_{v} g_{i}(\bar{u}, \bar{v}, 0)=\nabla_{p_{i}} h_{i}(\bar{u}, \bar{v}, 0)$, for all $i=\{1,2, \ldots, k\}$. 
(I) there exists $\bar{z} \in E$ such that $(\bar{u}, \bar{v}, \bar{\lambda}, \bar{z}, \bar{p}=0)$ is feasible for (WHP) and

(II) the objective values of (WHP) and (WHD) are equal.

Also, if the hypotheses of Theorem 3.1 are satisfied for all feasible solutions of (WHP) and (WHD), then $(\bar{u}, \bar{v}, \bar{\lambda}, \bar{z}, \bar{p}=0)$ is an efficient solution for (WHP).

Proof The proof follows along the lines of Theorem 3.2.

\section{Competing interests}

The authors declare that they have no competing interests.

\section{Authors' contributions}

All the authors contributed equally and significantly in writing this paper. All authors read and approved the final manuscript.

\section{Author details}

${ }^{1}$ Department of Mathematics, Indian Institute of Technology, Roorkee, 247667 , India. ${ }^{2}$ Faculty of Operations and Information Systems, Indian Institute of Management, Udaipur, 313 001, India.

\section{Acknowledgements}

The authors wish to thank the reviewer for her/his valuable and constructive suggestions, which have considerably improved the presentation of the paper. The first author is also grateful to the Ministry of Human Resource and Development, India for financial support to carry out this work.

Received: 22 September 2014 Accepted: 3 December 2014 Published: 06 Jan 2015

\section{References}

1. Chen, X: Higher-order symmetric duality in nondifferentiable multiobjective programming problems. J. Math. Anal. Appl. 290, 423-435 (2004)

2. Agarwal, RP, Ahmad, I, Gupta, SK: A note on higher-order nondifferentiable symmetric duality in multiobjective programming. Appl. Math. Lett. 24, 1308-1311 (2011)

3. Khurana, S: Symmetric duality in multiobjective programming involving generalized cone-invex functions. Eur. J. Oper. Res. 165, 592-597 (2005)

4. Kim, MH, Kim, DS: Nondifferentiable symmetric duality for multiobjective programming with cone constraints. Eur. J. Oper. Res. 188, 652-661 (2008)

5. Gupta, SK, Jayswal, A: Multiobjective higher-order symmetric duality involving generalized cone-invex functions. Comput. Math. Appl. 60, 3187-3192 (2010)

6. Gulati, TR, Gupta, SK: Higher-order symmetric duality with cone constraints. Appl. Math. Lett. 22, 776-781 (2009)

7. Gulati, TR, Mehndiratta, G: Nondifferentiable multiobjective Mond-Weir type second-order symmetric duality over cones. Optim. Lett. 4, 293-309 (2010)

8. Agarwal, RP, Ahmad, I, Jayswal, A: Higher-order symmetric duality in nondifferentiable multiobjective programming problems involving generalized cone convex functions. Math. Comput. Model. 52, 1644-1650 (2010)

9. Suneja, SK, Louhan, P: Higher-order symmetric duality under cone-invexity and other related concepts. J. Comput. Appl. Math. 255, 825-836 (2014)

10. Gupta, SK, Kailey, N, Kumar, S: Duality for nondifferentiable multiobjective higher-order symmetric programs over cones involving generalized $(F, \alpha, \rho, d)$-convexity. J. Inequal. Appl. 2012, 298 (2012)

11. Suneja, SK, Aggarwal, S, Davar, S: Multiobjective symmetric duality involving cones. Eur. J. Oper. Res. 141, 471-479 (2002)

10.1186/1029-242X-2015-3

Cite this article as: Debnath et al.: Symmetric duality for a higher-order nondifferentiable multiobjective

programming problem. Journal of Inequalities and Applications 2015, 2015:3 